\title{
Implications of 3-Dimensional Printed Spinal Implants on the Outcomes in Spine Surgery
}

\author{
Brian Fiani, ${ }_{1}^{1}$ Alexander Newhouse, ${ }^{2}$ Alessandra Cathel, ${ }^{1}$ Kasra Sarhadi, ${ }^{3}$ Marisol Soula ${ }^{4}$ \\ Department of Neurosurgery, Desert Regional Medical Center, Palm Springs, CA, USA \\ Department of Orthopedic Surgery, ${ }^{2}$ Rush University Medical Center, Chicago, IL, USA \\ Department of Neurology, ${ }^{3}$ University of Washington, Seattle, WA, USA \\ New York University School of Medicine, ${ }^{4}$ New York, NY, USA
}

Three-dimensional printing (3DP) applications possess substantial versatility within surgical applications, such as complex reconstructive surgeries and for the use of surgical resection guides. The capability of constructing an implant from a series of radiographic images to provide personalized anatomical fit is what makes 3D printed implants most appealing to surgeons. Our objective is to describe the process of integration of 3DP implants into the operating room for spinal surgery, summarize the outcomes of using 3DP implants in spinal surgery, and discuss the limitations and safety concerns during pre-operative consideration. 3DP allows for customized, light weight, and geometrically complex functional implants in spinal surgery in cases of decompression, tumor, and fusion. However, there are limitations such as the cost of the technology which is prohibitive to many hospitals. The novelty of this approach implies that the quantity of longitudinal studies is limited and our understanding of how the human body responds long term to these implants is still unclear. Although it has given surgeons the ability to improve outcomes, surgical strategies, and patient recovery, there is a need for prospective studies to follow the safety and efficacy of the usage of 3D printed implants in spine surgery.

Key Words: 3D printed · Spinal implants · Spine surgery · Innovation · Biologics.

\section{INTRODUCTION}

Three-dimensional printing (3DP) applications possess substantial versatility within surgical applications, such as complex reconstructive surgeries and for the use of surgical resection guides. The capability of constructing an implant from a series of radiographic images to provide personalized anatomical fit is what makes 3D printed implants most appealing to surgeons. The technology can be used in orthopedic sur- gery, neurological surgery, oral and maxillofacial surgery, plastic surgery, cardiothoracic surgery, and urologic surgery ${ }^{22)}$. The transition from "mass-produced" to "patient-specific 3DP implants" has sharpened surgical planning and reinvented the capabilities of surgical reconstruction that just a decade ago were extremely limited. The recent strides in improving magnetic resonance imaging (MRI), computed tomography (CT), and radiography benefit the accuracy of the precision through which anatomical structures can be reconstructed.

- Received : September 13, 2020 •Revised : October 14, 2020 •Accepted : December 7, 2020

- Address for reprints : Brian Fiani

Department of Neurosurgery, Desert Regional Medical Center, 1180 N. Indian Canyon Dr. Ste. 214 W Palm Springs, CA 92262, USA

Tel : +1-760-416-4511, Fax : +1-760-323-6201, E-mail : bfiani@outlook.com, ORCID : https://orcid.org/0000-0003-1700-4633

This is an Open Access article distributed under the terms of the Creative Commons Attribution Non-Commercial License (http://creativecommons.org/licenses/by-nc/4.0) which permits unrestricted non-commercial use, distribution, and reproduction in any medium, provided the original work is properly cited. 
Orthopedic surgery has long implemented 3DP into the operating room with personalized total hip arthroplasty to each patient specific anatomical nuance ${ }^{10)}$. While few, if any, manufacturers commercially advertise and produce patient-specific spinal implants, implementation of 3DP has chronologically transitioned from teaching instruments to custom surgical guides to patient tailored implants ${ }^{13)}$. With the intricate anatomy of the spine and the close proximity of neurovascular structures, the appeal to neurosurgeons and orthopedic surgeons when using 3D printed implants is the accuracy associated with the surgical procedure and the variety of spinal surgeries through which $3 \mathrm{DP}$ can be introduced to. Most 3D printed spinal implants have been reserved for complex oncologic reconstruction and traumatic or surgical defects and as these customized implants shift into elective spinal surgeries, patient reported outcomes are being explored on a case by case basis. Our objective is to describe the process of integration of 3DP implants into the operating room for spinal surgery, summarize the outcomes of using 3DP implants in spinal surgery, and discuss the limitations and safety concerns during pre-operative consideration.

\section{HISTORY OF 3DP IN MEDICINE/SURGERY}

For decades, physicians have been using computer aided design (CAD) software to build and visualize 3D representations of patients. These surgical templates have been used to understand an individual's unique anatomy and determine a surgical plan appropriate for that specific patient ${ }^{30)}$. In the realm of spine surgery, 3DP has been used to visualize the anatomy of the spine and its close relation to the surrounding neurovasculature to improve procedural accuracy ${ }^{30)}$. However, using this technology to physically replace human organs is a new concept and only recently being enthusiastically adopted into practice, especially in surgery.

In the 1990's 3DP was mostly used for dental implants and organ scaffolding. The first 3DP trials were pioneered by Dr. Anthony Atala. He printed customized scaffolds to grow human kidneys that were later implanted into patients. In the last decade, medical 3DP has evolved beyond scaffoldings ${ }^{3,39)}$. In 1999, D'Urso et al. ${ }^{11)}$, were the first in spine surgery to incorporate 3DP. Their study focused on using biomodelling via $3 \mathrm{D}$ printed technology to aid in complex surgeries. By visual- izing imaging data in a physical form, they were able to plan and rehearse surgery, navigate smoothly intraoperatively and better inform patients about surgical procedures ${ }^{11)}$. Within the last 5 years, 3DP in spine surgery has extended beyond preoperative surgical planning to the creation of patient-specific surgical instruments and the printing of omic implants). The first omic implants were led by Dr. Liu Zhongjun in 2014. He was the first surgeon to use 3DP to replace a 12-year-old's second vertebrae. His work has inspired similar surgeries worldwide because they confirmed that customized 3DP technology makes disc replacement stronger and more convenient than any other surgical approach. Novel and more recent applications include the creation of implantable 3D printed drug delivery systems. This idea is currently being tested in spinal tuberculosis where tuberculosis drugs are mixed with Poly-DLlactide and nano-hydroxyapatite and are delivered through an implant located in the spine ${ }^{30)}$.

Currently, 3DP allows for customized, light weight, and geometrically complex functional implants. It has given surgeons the ability to improve outcomes, surgical strategies, and patient recovery.

\section{BIOENGINEERING}

Introduction of a 3DP into the operating room requires extensive collaboration amongst physicians, engineers, and scientists. MRI and CT files of the patient's spine are imported into commercial segmentation software ${ }^{12,23)}$. A contrast threshold is manually chosen to facilitate bony segmentation and exclusion of surrounding soft tissue structures. Semi-automatic segmentation is performed to generate $3 \mathrm{D}$ printable stereolithography files and can be further imported into CAD software to achieve any post-processing manipulation, such as isolation of selective vertebrae ${ }^{4}$. Reconstruction of the bony morphology is necessary to visualize the defect or deformity so that fixation hardware or reconstructive anatomical structures can be fit appropriately. The design must consider form, function, fixation, and formation to achieve a reliable implant $^{15)}$. With a given defect, often times the construct is developed manually in CAD software and simulated in a finite element analysis (FEA) to determine expected biomechanics of the implant. Topological optimization (TO) is a novel FEA technique utilized to construct a minimum volume construct 
given a set of boundaries, such as support of loads in the sagittal, coronal, and horizontal planes ${ }^{32}$. Several studies utilize TO to provide a better understanding of the construct and whether it can withstand the biomechanical loads post-implantation $^{6,14,19)}$.

Upon construction of a design, material selection must consider biocompatibility, osseointegration, durability, and corrosion resistance. Titanium alloys are common material used in many spinal implants due to its stress-shielding properties and biocompatibility $^{40)}$. While implants can be printed with multiple materials, it can pose constraints due to the accessibility and cost of multiple-material 3D printers, requiring collaboration with manufacturers. Titanium alloys are printed using laser 3DP, with a common method being electron beam melting $^{277}$. Once printed, the implant must go through any postprinting processing such as polishing, threading of screw holes, and quality assurance ${ }^{33)}$. The printed implant can undergo additional biomechanical testing to ensure the loads simulated computationally are verified so that material failure is avoided. Because the implant is designed to a specific fit, a surgical guide can be printed to guide any surgical resections.

\section{BASIC BACKGROUND ON BIOMECHANICS OF THE SPINE AND HOW 3D IMPLANTS MAY IM- PACT}

The feasibility of 3D-printed patient-specific implants to function with proper spinal biomechanics is a very important consideration for this technology. Although the literature on customized implants utilized in spinal surgery is limited to a few case reports and case series, these examples demonstrated stability of the implants with minimal complications ${ }^{40)}$. This success was attributed to the ability to develop implants that deliver an impeccable fit specific to the patient's anatomy through precision printing. This customized fit is crucial when looking to develop implants that can distribute stress and shearing forces evenly and provide excellent osseointegration $^{13)}$. When vertebral body implants are surgically placed, the success of the implant relies heavily on the ability of its endplates to fit excellently with the vertebrae directly superior and inferior to it. Without this close fit allowing for the native bone to grow into and anchor the implant in place, the stability of that implant within the spine will more often than not be compromised, hindering the opportunity for proper spinal biomechanics going forward ${ }^{28)}$. For this reason, current spinal implant procedures are often equipped with implants of varying sizes and shapes to increase the chance for finding an optimal fit. Moreover, decisions to utilize bone grafts from other parts of the patient's body are also considered to attempt to provide the greatest likelihood for implant fit and success. However, this method requires extra procedures and thus is not without its own risk of complications. With the advent of 3DP to design highly-customized spinal implants, the issue of finding the perfect fit for patients would be superlatively addressed and would likely minimize both intraoperative and postoperative complications.

In addition to the question of fit, the material utilized to construct the spinal implant is also of vital importance in order to better ensure proper osseointegration of the implant. Current technologies aimed at designing vertebral body replacement as well as intervertebral disk implants focus on producing materials of a certain porosity to best match native bone stiffness and avoid stress-shielding effects ${ }^{28}$. Three materials have been largely used in this context, including titanium, polyetheretherketone (PEEK), and polycarbonate, all of which have been used to construct spinal implants that have demonstrated success in maintaining strength to withstand compressive forces without excessive stiffness ${ }^{28)}$. In addition, recent patents such as the $3 \mathrm{DP}$ spinal implants by $4 \mathrm{WEB}$ Medical are designed as webs that provide support along at least four planes of the implant and are proposed to enable bone growth and healing through a porous structure that allows for filling of the implant with graft material for supreme osseointegration $^{28)}$. Overall, the goals for optimal spinal implant design are focused around impeccable fit, high stresswithstanding materials, and seamless osseointegration, all of which appear more promising than ever with the ongoing research and development of 3DP spinal implants.

\section{APPLICATIONS FOR 3DP IN SPINE SURGERY}

\section{Oncologic disorders}

Outside of the use of 3DP implants as guides during spinal surgery, the majority of the literature on the use of 3DP implants focuses on oncological pathology. 3DP implants are valuable in visualizing the extent of tumor burden and the 
surrounding anatomy, especially relation to vital structures, which can aid the surgeon both pre- and intraoperatively during tumor resection planning and surgery (Table 1). Xiao et al. ${ }^{41)}$ discussed a series of patients who underwent en bloc resection in the cervical spine for primary malignant bone tumors. In this series, 3D models were used for preoperative planning. Similarly, Ahmed et al. ${ }^{1)}$, illustrated the valuable nature of 3D models. In this report, the model was used as reference intraoperatively to more readily appreciate the tumor and irregularity of lesions, so as to minimize morbidity; in addition, it aided in allowing the surgical team to obtain negative margins.

Many have utilized this technology to replace the deformity created after tumor excision. In 2016, Wei et al. ${ }^{38)}$ utilized 3DP status-post sacral chordoma resection, utilizing sacral replacement prosthesis. They found that at 8 months, there was asymptomatic instrumental failure and in-growth at bone-prosthesis interface. In 2016, Xu et al. ${ }^{42)}$, created a 3DP implant axial vertebral body replacement after surgery was undertaken for a C2 Ewing sarcoma. With this new technology, there were no complications noted from the surgical intervention, and no evidence of tumor re-growth, as well as satisfactory imaging at 1 year. With this customized implant, they were able to achieve a superior fit, as well as retain motion. Li et al. ${ }^{18)}$ printed a 3D implant for multilevel, C2-C4, artificial vertebral body, which was self-stabilizing, to reconstruct this region after metastatic papillary thyroid carcinoma resection. No subsidence was noted at 1 year. Kim et al. ${ }^{16)}$ in 2017 created a hemi-sacrum after sacral osteosarcoma resection. Again, no complications were noted post-operatively, with improved symptoms and imaging at 12-month follow up. Choy et al. ${ }^{8)}$ utilized an axial vertebral body device with inbuilt fixation holes for a T9 primary bone tumor, hemangioendothelioma. In this instance, the implant was fashioned with angled endplates so as to restore the sagittal balance. This patient was found to have improved symptoms, no complications, and imaging was acceptable at 6 months. Chin et al. ${ }^{7)}$, described a case report of recurrent giant cell tumor, which required en bloc spondylectomy of L1-L3. 3D reconstruction was able to fixate the posterior instrumentation, as well as allow for osseointegration. Mobbs et al. ${ }^{24)}$ also utilized the 3DP implant technology when operating for both a C1/2 chordoma and congenital L5 hemivertebra. In these cases, an occipto-cervical fixation device and hemivertebra prosthesis, respectively, were fashioned and though there was prolonged operation time in the first case, there were no imaging abnormalities at 9 and 12 months, respectively.

\section{Congenital disorders}

As demonstrated in the aforementioned case by Mobbs et al. ${ }^{24)}$, 3DP implants can also be utilized for congenital defects and/or deformities. In addition, biomodels can help plan for challenging parts of the procedure of these defects/deformities. Yang et al. ${ }^{43)}$ performed a retrospective study of 126 Lenke 1 adolescent idiopathic scoliosis patients. In this study, 3DP biomodels were compared to standard imaging; it was found that the 3D technology decreased operative time, blood loss, and transfusion volume with no difference in length of stay, complication rate, screw misalignment, or radiographic outcome. Moreover, in patients found to have a Cobb angle $>50$ degrees, it was found that utilizing the $3 \mathrm{D}$ model reduced screw misplacement significant $(p=0.02)$. In both adult and pediatric patients, 3DP implants have been implanted to aid in osteotomies. Tu et al. ${ }^{37)}$ reported a cohort of patients with ankylosing spondylitis resulting in severe kyphoscoliosis. These patients underwent software-aided simulated correction; osteotomy guides were printed and secured with pre-planned pedicle screw guides, resulting in 94\% accuracy of screw placement and no reported neurovascular complications.

\section{Degenerative disorders}

Degenerative disorders of the spine exist as highly prevalent pathologies that account for a large majority of chronic neck and back pain among patients of all ages. In fact, degenerative disk disease (DDD) is the most common diagnosis underlying back pain in the USA; a diagnosis that affects $40 \%$ of individuals younger than 30 and more than $90 \%$ of individuals over age $50^{2}$. Though classically defined as destruction brought on by stress-induced wear due to age-related changes or poor spinal biomechanics, spinal degeneration in general can occur due to a variety of etiologies, including invasive tumors and medication-induced osteopenia/osteoporosis. No matter what the etiology, the destructive changes within the spine that occur over time often leave patients with unique structural changes that would benefit most from unique structural solutions. In the event that surgery for vertebral/disk replacement or fixation is indicated, such as post-tumor resection, 3DP solutions would provide that specificity to maximize fit and os- 
Table 1. Summarization of literature regarding the implementation of 3DP into spine surgery practice stratified by category and year

\begin{tabular}{|c|c|c|}
\hline Study & Classification & Summarization \\
\hline Kim et al. ${ }^{16)}(2017)$ & Oncologic & Created a hemi-sacrum after sacral osteosarcoma resection; no complications; improved symptoms. \\
\hline Xiao et al. ${ }^{411}(2016)$ & Oncologic & aluable in pre-operative planning; visualizing the extent of tumor burden and the surrounding anatomy. \\
\hline Xu et al. ${ }^{42)}(2016)$ & Oncologic & $\begin{array}{l}\text { Vertebral body replacement after surgery was undertaken for a C2 Ewing sarcoma; no complications; } \\
\text { superior fit. }\end{array}$ \\
\hline Wei et al. ${ }^{38)}(2017)$ & Oncologic & $\begin{array}{l}\text { Sacral replacement prosthesis status-post sacral chordoma resection; asymptomatic instrumental failure } \\
\text { and in-growth at bone-prosthesis interface. }\end{array}$ \\
\hline Choy et al. $^{8)}(2017)$ & Oncologic & $\begin{array}{l}\text { Utilized an axial vertebral body device with inbuilt fixation holes and angled endplates for a T9 primary } \\
\text { bone tumor; restored sagittal balance; improved symptoms. }\end{array}$ \\
\hline Li et al. ${ }^{18)}(2017)$ & Oncologic & $\begin{array}{l}\text { Multilevel, C2-C4, artificial vertebral body, which was self-stabilizing, to reconstruct this region after meta- } \\
\text { static papillary thyroid carcinoma resection. No subsidence was noted at } 1 \text { year. }\end{array}$ \\
\hline s et al & $\begin{array}{l}\text { Oncologic/ } \\
\text { congenital }\end{array}$ & $\begin{array}{l}\mathrm{C} 1 / 2 \text { chordoma and congenital L5 hemivertebra. In these cases, an occipto-cervical fixation device and } \\
\text { hemivertebra prosthesis, respectively, were fashioned and though there was prolonged operation time } \\
\text { in the first case, there were no imaging abnormalities at } 9 \text { and } 12 \text { months, respectively. }\end{array}$ \\
\hline Chin et al. ${ }^{7)}$ (2019) & Oncologic & $\begin{array}{l}3 \mathrm{D} \text { reconstruction was able to fixate the posterior instrumentation, as well as allow for osseointegration } \\
\text { status-post en bloc spondylectomy of } \mathrm{L} 1-\mathrm{L} 3 \text { recurrent giant cell tumor. }\end{array}$ \\
\hline Ahmed et al.' (2019) & Oncologic & $\begin{array}{l}\text { Referenced intraoperatively to more readily appreciate the tumor and irregularity of lesions, so as to } \\
\text { minimize morbidity; in addition, it aided in allowing the surgical team to obtain negative margins. }\end{array}$ \\
\hline Yang et al. ${ }^{43)}$ (2015) & Congenital & $\begin{array}{l}\text { 3D technology decreased operative time, blood loss, and transfusion volume with no difference in LOS, } \\
\text { complication rate, screw misalignment, or radiographic outcome. Moreover, in patients found to have } \\
\text { a Cobb angle }>50 \text { degrees, it was found that utilizing the 3D model reduced screw misplacement } \\
\text { significant ( } p=0.02 \text { ). }\end{array}$ \\
\hline al. ${ }^{37)}(2019)$ & Cor & $\begin{array}{l}\text { Software-aided simulated correction; } 94 \% \text { accuracy of screw placement and no reported neurovascular } \\
\text { complications }\end{array}$ \\
\hline $\begin{array}{l}\text { Rosenzweig et al. }{ }^{31)} \\
\text { (2015) }\end{array}$ & & $\begin{array}{l}\text { In vitro experiments showed that primary articular chondrocytes and nucleus pulposus cells could be } \\
\text { cultured successfully on 3D-printed acrylonitrile butadiene styrene and polylactic acid scaffolds for } \\
\text { osseointegration. }\end{array}$ \\
\hline Siu et al. ${ }^{33)}$ (2018) & Degenerative & $\begin{array}{l}\text { Customized cages for lumbar radiculopathy and osteoporosis; resolution of symptoms at } 6 \text { month follow } \\
\text { up and CT demonstrated fusion. }\end{array}$ \\
\hline Lu et al. ${ }^{21)}$ (2017) & $\begin{array}{l}\text { Single/ } \\
\text { multi-level fusion }\end{array}$ & $\begin{array}{l}\text { 3DP titanium fusion cage was used for single-level anterior cervical corpectomy and fusion in } 15 \text { patients } \\
\text { with cervical spondylotic myelopathy and ossification of the posterior longitudinal ligament; solid } \\
\text { interbody fusion and relief from pain and neurological symptoms in all patients. }\end{array}$ \\
\hline $\begin{array}{l}\text { Thayaparan et al. }{ }^{36)} \\
\text { (2018) }\end{array}$ & $\begin{array}{l}\text { Single/ } \\
\text { multi-level fusion }\end{array}$ & $\begin{array}{l}\text { 3DP patient-specific titanium atlantoaxial screws were used. Follow-up at one month, } 2 \text { months, and } 6 \\
\text { months demonstrated successful screw placement and fixation assessed via CT imaging, along with } \\
\text { no neurological problems, readmissions, or instances of implant failure in any of the patients at the } \\
\text { 12-month post-operative mark. }\end{array}$ \\
\hline $\begin{array}{l}\text { Mokawem et al. }{ }^{26)} \\
(2019)\end{array}$ & $\begin{array}{l}\text { Single/ } \\
\text { multi-level fusion }\end{array}$ & $\begin{array}{l}\text { Transforaminal lumbar interbody fusion or lateral lumbar interbody fusion with silicate-substituted calcium } \\
\text { phosphate-packed 3DP lamellar titanium cages printed from 3D reconstructions of CT images of each } \\
\text { patient demonstrated excellent fusion success in } 92 \text { of the } 93 \text { patients ( } 98.9 \% \text { success rate), as well as } \\
\text { significantly improved patient-reported outcomes/satisfaction. }\end{array}$ \\
\hline Ling et al. ${ }^{20)}$ (2018) & $\begin{array}{l}\text { Spinal } \\
\text { decompression }\end{array}$ & $\begin{array}{l}\text { Utilized 3DP models in the pre-operative planning of a "V"-shaped decompressive laminoplasty for } \\
\text { multilevel ossification of the ligamentum flavum; successful decompression. }\end{array}$ \\
\hline Choy et al. ${ }^{9)}$ (2018) & $\begin{array}{l}\text { Spinal } \\
\text { decompression }\end{array}$ & $\begin{array}{l}\text { Successful anterior cervical decompression and multi-level fusion with patient-specific 3DP implants made } \\
\text { of titanium alloy in challenging deformity setting. }\end{array}$ \\
\hline Mobbs et al. ${ }^{25)}$ (201 & $\begin{array}{l}\text { Spinal } \\
\text { decompression }\end{array}$ & $\begin{array}{l}\text { 3DP technology in anterior lumbar interbody fusion surgery; superb fit, but also as tools for pre-operative } \\
\text { planning and practice; restored proper lumbar lordosis; clinical improvement; reduced operative time. }\end{array}$ \\
\hline
\end{tabular}

3DP : three-dimensional printing, LOS : length of stay, CT : computed tomography 
seointegration necessary for post-operative success and improved quality of life for patients.

Although the literature demonstrating the potential of 3DP spinal implants for degenerative disorders of the spine is limited to case reports and theoretical experiments, there are promising results within these initial findings. For example, a case report published by Siu et al. ${ }^{33)}$ detailed a 74 -year old woman with L2-L3 osteoporotic fractures who received 3Dprinted implantable titanium cages with remarkable success in implant-end plate matching and restoration of lost disk space. This patient was able to ambulate from postoperative day 1 and experienced resolution of radicular symptoms with CT evidence of successful fusion at 6-month follow-up. In the theoretical realm, Rosenzweig et al. ${ }^{31)}$ demonstrated through in vitro experiments that primary articular chondrocytes and nucleus pulposus cells could be cultured successfully on 3Dprinted acrylonitrile butadiene styrene and polylactic acid scaffolds. These in vitro successes illustrate that 3D-printed implants have the potential for successful osseointegration through the promotion of both bony and soft tissue growth and restoration. As the population continues to age and degenerative disorders of the spine continue to grow in prevalence, more research into 3DP spinal implants is needed to build on these initial successes that show the noteworthy advantage of customizable and readily-producible implantable solutions.

\section{Single/multi-level fusions}

Spinal fusion surgeries play a primary role in correcting problems like spondylosis and bony deformities that can manifest as myelopathy and radiculopathy by fusing adjacent vertebrae to provide improved stability and alignment. These surgeries typically make use of industry-produced intervertebral fusion cages made of either PEEK or titanium of various sizes which are then evaluated by the surgical team and chosen on what appears to fit best with a patient's anatomy. This standard approach allows for a high rate of success through utilizing porous material that allows for native bone to grow into and throughout the cage, effectively making the fusion cage part of the patient's spine.

As mentioned previously, improving the probability of successful osseointegration relies on various factors, one of which is producing surgical material with an impeccable fit tailored to each individual patient. Much like other spinal surgeries, spinal fusion is a procedure that has shown promise in utilizing 3DP fusion cages for improved surgical outcomes in both single- and multi-level fusions. In a retrospective analysis completed by Mokawem et al. ${ }^{26)}$ in the UK, a consecutive series of 93 patients with lumbar degenerative disease or deformity requiring interbody fusion cages underwent transforaminal lumbar interbody fusion or lateral lumbar interbody fusion with silicate-substituted calcium phosphate-packed 3DP lamellar titanium cages printed from 3D reconstructions of CT images of each patient. After evaluating the solidity of fusion 12 months after surgery and concurrently administering surveys assessing patient quality-of-life, pain, and level of disability, the authors demonstrated excellent fusion success in 92 of the 93 patients (98.9\% success rate), as well as significantly improved patient-reported outcomes/satisfaction, demonstrating superb success with this 3DP fusion cage approach. In another retrospective case series study completed by Lu et al. ${ }^{21)}$ from China, a 3DP titanium fusion cage was used for single-level anterior cervical corpectomy and fusion in 15 patients with cervical spondylotic myelopathy and ossification of the posterior longitudinal ligament. Outcomes for this study included 6-month post-operative assessment of fusion success through evaluation of interbody angle and height and surveys assessing patient functional neurological status and pain. Results demonstrated both solid interbody fusion and relief from pain and neurological symptoms in all patients, illustrating remarkable success with this 3DP anatomy-adaptive titanium mesh cage for single-level anterior cervical corpectomy and fusion in patients with cervical spondylotic myelopathy and ossification of the posterior longitudinal ligament.

As an alternative to spinal fusion cages, another spinal fusion technique that lends itself to 3DP device adaptation is fusion utilizing transarticular screws, such as in atlantoaxial transarticular screw fixation for arthrodesis. A study completed by Thayaparan et al. ${ }^{36)}$ from Australia reports the successful use of 3DP patient-specific titanium screws manufactured to best fit with 3DP models of each patient's atlantoaxial spine from $3 \mathrm{D}$ reconstructions of CT imaging. This specific fit is highly beneficial in the case of transarticular atlantoaxial screw fixation given the nontrivial risk of iatrogenic neurovascular injury. In this case series, the screws were placed transarticularly and along the posterior $\mathrm{Cl}$ body arch with no screw malposition or neurovascular injury observed in any of the patients. Follow-up at 1 month, 2 months, and 6 months dem- 
onstrated successful screw placement and fixation assessed via CT imaging, along with no neurological problems, readmissions, or instances of implant failure in any of the patients at the 12-month post-operative mark ${ }^{36)}$.

Currently, the literature supporting the use of 3DP spinal implants in patients requiring single- or multi-level spinal fusion demonstrate that both spinal fusion cages and screws are two viable 3DP options that can provide incredible surgical outcomes and patient satisfaction. As 3DP spinal implants become more available and cost-effective to produce, randomized-controlled trials comparing their success with standard options will be able to shed more light on whether this advanced technology warrants the investment compared to current standards of spinal implants.

\section{Spinal decompression}

With most spinal surgical interventions, the goal is to decompress any neural structures currently impacted by the present pathology and to implement a solution that is structurally stable, functioning, and minimizes the chance that these neural structures are re-compressed in the future. The current literature on 3DP implants in spinal surgery demonstrates the successful use of utilizing implants in both decompressive and reconstructive phases of surgery, typically in interbody fusion procedures ${ }^{17)}$. Even though this data is limited, a systematic review authored by Choy et al. regarding the use of 3DP implants in anterior cervical surgery identified two case reports of patients undergoing successful anterior cervical decompression and multi-level fusion with patient-specific 3DP implants made of titanium alloy ${ }^{9,34)}$. While standardlyproduced implants are also successful in achieving good outcomes in these types of procedures, the benefit of 3DP implants was truly shown in these two patients with very surgically-challenging deformities caused by malignancy affecting the anatomically-unique axis (C2 vertebra $)^{9)}$.

Moreover, in a case report published by Mobbs et al. ${ }^{25}$, the authors presented the successful use of 3DP technology in anterior lumbar interbody fusion surgery in a 34-year old male with bilateral L5 radiculopathy caused by a bilateral L5 pars defect, L5/S1 DDD and severe foraminal stenosis. This case report was unique in that it demonstrated the high value of patient-specific 3DP models not only as the basis of an implant with superb fit, but also as tools for pre-operative planning and practice. From the patient's CT imaging, the authors produced 3DP models that achieved the goal of restoring proper lumbar lordosis and provided the opportunity to preplan screw holes in the implant. This 3DP approach was beneficial in both producing profound clinical improvement in the patient as well as reducing operative time with improved preoperative planning. A separate case report published by Ling et al. ${ }^{20)}$ also demonstrated the benefit of 3DP models in surgical planning of spinal decompression. In their 66-year old patient, they utilized 3DP models in the pre-operative planning of a "V"-shaped decompressive laminoplasty for multilevel ossification of the ligamentum flavum. With no reported surgical complications and successful decompression and relief of the patient's clinical symptoms, these authors demonstrated the value of 3DP models for planning of spinal interventions.

Overall, although use of 3DP models and implants in spinal decompression procedures is still growing, the early reports show great promise. Through facilitating both precise planning and a smooth intraoperative experience, these patientspecific implants are slowly but surely establishing a strong foothold in the world of spinal surgery.

\section{LIMITATIONS OF 3D PRINTED IMPLANTS IN SPINE SURGERY}

Although a promising technology, 3DP has some limitations that affect its routine use in spine surgical practice. 3DP is common, but not universal. As a result, not every facility has access to printers, technicians, and trained physicians. Additionally, the cost of this technology is prohibitive to many hospitals.

Even if a facility has access to 3D printers, the technology itself still poses some barriers. Printing speed, resolution and reproducibility may be far from optimal ${ }^{29)}$. Most of the $3 \mathrm{D}$ prints used for spine surgery can take anywhere from 10-12 hours to design and print. This burden of time can vary exponentially depending on a user's comfort with prototype softwares used for designing. These softwares are notoriously known to have steep learning curves. For standard procedures like decompression surgery, the cost of printing cannot be justified for the potential difference in operation times and success rate. This is a deterrent for many cases as the preoperative time consumption exceeds any currently known potential benefits ${ }^{13)}$. There exists a few scenarios where the long produc- 
tion times and the high financial costs and time-related burden of 3D technology is justified. Usually these are niche, variable and complex surgeries like pediatrics spinal deformities and spinal tumor resections ${ }^{13)}$.

Additionally, sufficient biocompatible materials that can be safely and chronically implanted into patients do not exist. The novelty of this approach implies that the quantity of longitudinal studies is limited and our understanding of how the human body responds long term to these implants is still unclear. Biomaterials that exhibit tissue biomimicry and can regulate inflammation, fracture healing, and bone microenvironment need to be further researched. Lastly, as any emerging technology, the United States Food and Drug Administration is attempting to create a protocol for approving 3D printed implants and safe protocols for their use in human patients. These regulations have yet to be fully developed and as a result has been limiting the use of the technology in healthcare settings ${ }^{29)}$.

\section{SAFETY AND EFFICACY}

Prior to constructing the 3DP implants, multiple considerations must be discussed to determine the necessary requirements to verify the efficacy in implanting a custom-made construct. Because these endeavors are categorized as explorative, guidelines can be stringent to minimize the risk involved $^{20)}$. The argument for 3DP implants can gain approval in complex defects and traumatic anatomical destruction where commercial implants will not suffice. While multiple studies discuss the implementation of 3DP implants in spine surgery, there is a lack of clarity on the associated legal process $^{22,35)}$. When considering 3DP patient specific implants, a comprehensive framework with established evidence for use must be presented to the International Review Board to ensure its efficacy. Surgical advantages with 3DP implants include precision of implant shape, reduced operating room time and incidence of complications, and improved patient outcomes, while disadvantages entail additional extensive preoperative preparation, minor inaccuracies of the computer software and 3D printer, and cost of all resources ${ }^{22)}$. To mitigate complications and risk of error, surgical trials with the 3DP implant in cadavers are suggested to identify any unforeseen obstacles. While there are recommended indications for the use of 3DP implants in segmental defects in lower extremity orthopedic surgery, a similar proposal is needed to guide indications in spine pathology ${ }^{35)}$.

\section{PREOPERATIVE CONSIDERATIONS}

Much is yet to be known in regards to 3DP implants in spinal surgery. While there is literature that has shown its benefit in regards to pre-operative planning and utilization as guides, the research on implementation as prosthesis is much less described. Functioning as a guide, 3DP implants have demonstrated improved accuracy of screw placement and allow surgeons to account for anatomical structures that may otherwise have been damaged. Moreover, the use of a 3D model guides allows for surgeons who may feel less than ideally comfortable in an operation to feel more reassured in regards to trajectory, placement, and overall surgical execution.

In regards to implantation, this technology lends to a more customized fit, leading to better ossification, less chance of subsidence, preserved motion for some, and ability to conform the implant to the patient's individual anatomy. As abovementioned, it also allows for the surgeon to visualize the full scope of the anatomy, whether for a tumor or a defect. Therefore, patient selection for 3DP implants should be those patients with challenging anatomy, secondary to deformity, fracture, tumor, or iatrogenic cause.

Most implants are fashioned from titanium, which is commonplace presently in spinal implants. However, care must be taken to explain to patients, once deemed candidates, that use for implantation is still novel, and allow them to weigh the risks of utilization. In addition, the cost of the implant can result in difficulty proceeding via this route when compared with standard shelf implants. Risks of the individual intended surgical procedure need to be reviewed in detail with the patient, as is usual practice with any neurosurgical intervention.

\section{CONCLUSION}

In the present paper, we explore the existing applications of 3DP implants in spine surgery, their respective outcomes, and the considerations that are discussed when using patient specific interventions. In the last decade, an increasing number of case series in the literature utilizing 3DP implants in both 
complex reconstructions and elective spine surgeries have yielded promising outcomes. Achieving superior outcomes is highly dependent on the collaboration of physicians, scientists, and engineers to establish a thorough workflow to yield an optimal outcome. In elective surgeries, surgeons can be deterred by the extensive process involved with using 3DP implants and the unforeseen outcomes compared to using standard implants. Gathering a better understanding of the longterm reliability and outcomes of these implants is imperative to establish them as viable options for patients undergoing spine surgery.

\section{CONFLICTS OF INTEREST}

No potential conflict of interest relevant to this article was reported.

\section{INFORMED CONSENT}

This type of study does not require informed consent.

\section{AUTHOR CONTRIBUTIONS}

\author{
Conceptualization : BF \\ Data curation : $\mathrm{BF}$ \\ Formal analysis : BF \\ Methodology : BF \\ Project administration : $\mathrm{BF}$ \\ Visualization : BF \\ Writing - original draft : BF, AN, AC, KS, MS \\ Writing - review \& editing: $\mathrm{BF}$
}

\section{ORCID}

Brian Fiani https://orcid.org/0000-0003-1700-4633

Alexander Newhouse

Alessandra Cathel https://orcid.org/0000-0002-7751-5251

Kasra Sarhadi https://orcid.org/0000-0001-7013-6999

Marisol Soula https://orcid.org/0000-0001-9278-6191 https://orcid.org/0000-0002-3326-5019

\section{References}

1. Ahmed AK, Pennington Z, Molina CA, Xia Y, Goodwin CR, Sciubba DM : Multidisciplinary surgical planning for en bloc resection of malignant primary cervical spine tumors involving 3D-printed models and neoadjuvant therapies: report of 2 cases. J Neurosurg Spine 30 : 417-550, 2019

2. Andersson GB : Epidemiological features of chronic low-back pain. Lancet $354:$ 581-585, 1999

3. Atala $A$, Forgacs $G$ : Three-dimensional bioprinting in regenerative medicine: reality, hype, and future. Stem Cells Transl Med 8 : 744745, 2019

4. Autodesk Inc. : Meshmixer. Available at : https://www.autodesk.com/ research/projects/meshmixer?us_oa=dotcom-us\&us_si=750abd7ee5de-4fbe-bc5b-1174514b7afe\&us_st=Meshmixer

5. Burleson J, DiPaola C : 3D printing in spine surgery in Dipaola $M$, Wodajo FM (eds) : 3D Printing in Orthopaedic Surgery, ed 1. Philadelphia : Elsevier, 2019, pp105-122

6. Chen CS, Shih SL: Biomechanical analysis of a new lumbar interspinous device with optimized topology. Med Biol Eng Comput 56 : 1333 1341, 2018

7. Chin BZ, Ji T, Tang X, Yang R, Guo W : Three-level lumbar en bloc spondylectomy with three-dimensional-printed vertebrae reconstruction for recurrent giant cell tumor. World Neurosurg 129 : 531-537.e1, 2019

8. Choy WJ, Mobbs RJ, Wilcox B, Phan S, Phan K, Sutterlin CE 3rd : Reconstruction of thoracic spine using a personalized 3D-printed vertebral body in adolescent with T9 primary bone tumor. World Neurosurg 105 : 1032.e13-1032.e17, 2017

9. Choy WJ, Parr WCH, Phan K, Walsh WR, Mobbs RJ : 3-dimensional printing for anterior cervical surgery: a review. J Spine Surg 4 : $757-$ 769, 2018

10. Conformis : Conformis hip system. Available at : https://www.conformis.com/conformis-hip-system/

11. D'Urso PS, Askin G, Earwaker JS, Merry GS, Thompson RG, Barker TM, et al. : Spinal biomodeling. Spine (Phila Pa 1976) 24 : 1247-1251, 1999

12. Fedorov A, Beichel R, Kalpathy-Cramer J, Finet J, Fillion-Robin JC, Pujol $S$, et al. : 3D Slicer as an image computing platform for the Quantitative Imaging Network. Magn Reson Imaging 30 : 1323-1341, 2012

13. Garg B, Mehta N : Current status of 3D printing in spine surgery. J Clin Orthop Trauma $9:$ 218-225, 2018

14. Guo LX, Yin JY : Finite element analysis and design of an interspinous device using topology optimization. Med Biol Eng Comput 57 : 89 98, 2019

15. Hollister SJ : Scaffold design and manufacturing: from concept to clinic. Adv Mater 21 : 3330-3342, 2009

16. Kim D, Lim JY, Shim KW, Han JW, Yi S, Yoon DH, et al. : Sacral reconstruction with a 3D-printed implant after hemisacrectomy in a patient with sacral osteosarcoma: 1-year follow-up result. Yonsei Med J 58 : 453-457, 2017

17. Landi A, Delfini R, Ricci A, Barbanera A, Anichini G, Brogna C : New 
trends in instrumentation and complex techniques in spine surgery. Biomed Res Int 2015 : 216384, 2015

18. Li X, Wang Y, Zhao Y, Liu J, Xiao S, Mao K : Multilevel 3D printing implant for reconstructing cervical spine with metastatic papillary thyroid carcinoma. Spine (Phila Pa 1976) 42 : E1326-E1330, 2017

19. Lin HM, Liu CL, Pan YN, Huang CH, Shih SL, Wei SH, et al. : Biomechanical analysis and design of a dynamic spinal fixator using topology optimization: a finite element analysis. Med Biol Eng Comput 52 : 499508, 2014

20. Ling Q, He E, Ouyang H, Guo J, Yin Z, Huang W : Design of mulitlevel OLF approach ("V"-shaped decompressive laminoplasty) based on 3D printing technology. Eur Spine J 27(Suppl 3) : 323-329, 2018

21. Lu T, Liu C, Yang B, Liu J, Zhang F, Wang D, et al. : Single-level anterior cervical corpectomy and fusion using a new 3D-printed anatomyadaptive titanium mesh cage for treatment of cervical spondylotic myelopathy and ossification of the posterior longitudinal ligament: a retrospective case series study. Med Sci Monit 23 : 3105-3114, 2017

22. Martelli N, Serrano C, van den Brink $H$, Pineau J, Prognon P, Borget I, et al. : Advantages and disadvantages of 3-dimensional printing in surgery: a systematic review. Surgery 159 : 1485-1500, 2016

23. Materialise : Always There. Empowering your 3D printing applications. Available at : https://www.materialise.com/

24. Mobbs RJ, Coughlan M, Thompson R, Sutterlin CE 3rd, Phan K: The utility of $3 \mathrm{D}$ printing for surgical planning and patient-specific implant design for complex spinal pathologies: case report. J Neurosurg Spine $26: 513-518,2017$

25. Mobbs RJ, Parr WCH, Choy WJ, McEvoy A, Walsh WR, Phan K : Anterior lumbar interbody fusion using a personalized approach : is custom the future of implants for anterior lumbar interbody fusion surgery? World Neurosurg 124 : 452-458.e1, 2019

26. Mokawem M, Katzouraki G, Harman CL, Lee R : Lumbar interbody fusion rates with 3D-printed lamellar titanium cages using a silicatesubstituted calcium phosphate bone graft. J Clin Neurosci 68 : 134139, 2019

27. Popov VV Jr, Muller-Kamskii G, Kovalevsky A, Dzhenzhera G, Strokin E, Kolomiets $\mathrm{A}$, et al. : Design and 3D-printing of titanium bone implants: brief review of approach and clinical cases. Biomed Eng Lett 8 : 337344,2018

28. Provaggi E, Leong JJH, Kalaskar DM : Applications of $3 \mathrm{D}$ printing in the management of severe spinal conditions. Proc Inst Mech Eng H 231 : 471-486, 2017

29. Pucci JU, Christophe BR, Sisti JA, Connolly ES Jr : Three-dimensional printing: technologies, applications, and limitations in neurosurgery. Biotechnol Adv 35 : 521-529, 2017

30. Roopavath UK, Kalaskar DM : Introduction to 3D printing in medicine in
Kalaskar DM (ed) : 3D Printing in Medicine, ed 1. Woodhead Publishing: Cambridge, 2017, pp1-20

31. Rosenzweig DH, Carelli E, Steffen T, Jarzem P, Haglund L : 3D-printed ABS and PLA scaffolds for cartilage and nucleus pulposus tissue regeneration. Int J Mol Sci 16 : 15118-15135, 2015

32. Sigmund 0 : Topology optimization: a tool for the tailoring of structures and materials. Philos Trans R Soc Lond A 358 : 211-227, 2000

33. Siu $T L$, Rogers JM, Lin $K$, Thompson R, Owbridge $M$ : Custom-made titanium 3-dimensional printed interbody cages for treatment of osteoporotic fracture-related spinal deformity. World Neurosurg $111: 1-5$, 2018

34. Spetzger U, Frasca M, König SA : Surgical planning, manufacturing and implantation of an individualized cervical fusion titanium cage using patient-specific data. Eur Spine J 25 : 2239-2246, 2016

35. Tetsworth K, Block S, Glatt V : Putting 3D modelling and 3D printing into practice: virtual surgery and preoperative planning to reconstruct complex post-traumatic skeletal deformities and defects. SICOT J 3 : 16, 2017

36. Thayaparan GK, Owbridge MG, Thompson RG, D'Urso PS : Designing patient-specific 3D printed devices for posterior atlantoaxial transarticular fixation surgery. J Clin Neurosci 56 : 192-198, 2018

37. Tu Q, Ding HW, Chen H, Miao QJ, Yang X, Li K, et al. : Three-dimensional-printed individualized guiding templates for surgical correction of severe kyphoscoliosis secondary to ankylosing spondylitis: outcomes of 9 cases. World Neurosurg 130 : e961-e970, 2019

38. Wei R, Guo W, Ji T, Zhang Y, Liang H : One-step reconstruction with a 3D-printed, custom-made prosthesis after total en bloc sacrectomy: a technical note. Eur Spine J 26 : 1902-1909, 2017

39. Whitaker $\mathrm{M}$ : The history of $3 \mathrm{D}$ printing in healthcare. The Bulletin of the Royal College of Surgeons of England 96 : 228-229, 2014

40. Wilcox B, Mobbs RJ, Wu AM, Phan K : Systematic review of 3D printing in spinal surgery: the current state of play. J Spine Surg 3 : 433-443, 2017

41. Xiao JR, Huang WD, Yang XH, Yan WJ, Song DW, Wei HF, et al. : En bloc resection of primary malignant bone tumor in the cervical spine based on 3-dimensional printing technology. Orthop Surg 8 : 171-178, 2016

42. Xu N, Wei F, Liu X, Jiang L, Cai H, Li Z, et al. : Reconstruction of the upper cervical spine using a personalized 3D-printed vertebral body in an adolescent with Ewing sarcoma. Spine (Phila Pa 1976) 41 : E50-E54, 2016

43. Yang M, Li C, Li Y, Zhao Y, Wei X, Zhang G, et al. : Application of 3D rapid prototyping technology in posterior corrective surgery for Lenke 1 adolescent idiopathic scoliosis patients. Medicine (Baltimore) 94 : e582, 2015 